\title{
La paz y la solución al problema del campo en Colombia: un análisis comparado entre el Acuerdo de Paz y el Plan Nacional de Desarrollo
}

\section{Peace and the Solution to the Problem of the Field in Colombia: A Comparative Analysis in the Peace Agreement and the National Development Plan}

\author{
Eduardo Sánchez Iglesias ${ }^{1}$ \\ Vicente Sánchez Jiménez ${ }^{2}$ \\ Fundación Hábitat / Universidad Complutense de \\ Madrid (España)
}

Recibido: 11-10-17

Aprobado: 19-01-18

\section{Resumen}

El debate sobre los diferentes escenarios que se abren dentro del posconflicto colombiano remiten a un aspecto central: la solución del problema del acceso de la tierra y la superación de las brechas campo/ciudad. La hipótesis que plantea el presente trabajo entiende que el éxito del posconflicto en Colombia reside, en una parte importante, en la puesta en práctica de una política por parte del Gobierno Nacional que guarde relación con la solución al problema campo previsto en el Acuerdo de paz. El presente texto pretende realizar análisis comparativos entre las medidas para la transformación del campo colombiano contempladas en el Acuerdo de paz y el Plan Nacional de Desarrollo del Gobierno Nacional, principales documentos donde se abordan dicha problemática.

Palabras-clave: Acuerdo de Paz, reforma agraria, política económica, desarrollo, Colombia.

\footnotetext{
${ }^{1}$ (eduardo.sanchez@invi.uned.es). Doctor en Economía Internacional y Desarrollo de la Universidad Complutense de Madrid y Profesor Colaborador de Ciencias Políticas en la UNED, España.

2 (vsanchez@fecoma.ccoo.es). Doctor en Economía Aplicada de la Universidad Complutense de Madrid y Presidente de la Fundación Hábitat: entorno, economía y sociedad, España.
} 


\begin{abstract}
The debate on the different scenarios that open up in the post-conflict Colombia refer to a central aspect: the solution of the problem of access to land overcoming of the field/city gaps. The hypothesis that the present work proposes, understands that the success of the post-conflict in Colombia resides, in an important part, in the implementation of a policy by the National Government that is related to the solution to the problem area foressen in the Agreement of peace. The present text intends to carry out comparative analyzes between the measures for the transformation of the Colombian countryside contemplated in the Peace Agreement and the National Development Plan of the National Government, the main documents where this problem is addressed.
\end{abstract}

Key-words: Agreement of Peace, Agrarian Reform, Economic Policy, Development, Colombia.

\title{
1. Introducción
}

El debate sobre los diferentes escenarios que se abren dentro del posconflicto colombiano, descansan en gran parte en las condiciones económicas y sociales que puedan hacer posibles los "derechos políticos, sociales, económicos y culturales" previstos en el Acuerdo final (2016: 2), los cuales remiten a un aspecto central: la solución del problema del acceso de la tierra y la superación de las brechas campo/ciudad. El presente texto pretende realizar análisis comparativos entre las medidas para la transformación del campo colombiano contempladas en el Acuerdo de paz y el Plan Nacional de Desarrollo del Gobierno Nacional (PND), principales documentos donde se abordan dicha problemática.

La hipótesis que plantea el presente trabajo, entiende que el éxito del posconflicto en Colombia reside, en una parte importante, en la puesta en práctica de una política por parte del Gobierno Nacional que guarde relación con la solución al problema campo previsto en el Acuerdo de paz. Esta consideración obliga a estudiar la relación existente entre los principios y acciones que debe seguir ese "nuevo paradigma de desarrollo y bienestar" contemplado en el Acuerdo de paz y el modelo de desarrollo defendido por el Gobierno Nacional, en cuanto que principal actor responsable en la puesta en práctica del contenido económico descrito en el texto del Acuerdo final.

Dicho Acuerdo final define de manera expresa que la "meta esencia de la reconciliación nacional [reside en] la construcción de un nuevo paradigma de desarrollo y bienestar territorial para beneficio de amplios sectores de la población hasta ahora víctima de la exclusión y la desesperanza" (2016: 3). De 
esta forma se vinculan el logro de una paz duradera y definitiva, con la puesta en práctica de un modelo de desarrollo que tiene en la dimensión económica y social sus aspectos centrales.

Se procederá a realizar un análisis de las principales medidas económicas y sociales incluidas en el Acuerdo final respecto a la reforma rural integral, para posteriormente estudiar el Plan Nacional de Desarrollo 2014-2018, documento en el que se expone la política económica y social del Gobierno Nacional, para terminar con un apartado de conclusiones en las que abordar el grado de relación y coherencia existente entre lo previsto en el Acuerdo de paz y la política económica planteada desde el Gobierno Nacional y la relación que pueda tener con el escenario de posconflicto armado en Colombia.

\section{Principios generales del Acuerdo final}

Después de más de medio siglo de enfrentamiento armado entre el Gobierno colombiano y las Fuerzas Armadas Revolucionarias de ColombiaEjército del Pueblo (FARC-EP), el 26 de noviembre de 2016 se suscribió en Bogotá el Acuerdo Final para la Terminación del Conflicto y Construcción de la Paz Estable y Duradera, que puso fin al conflicto armado más antiguo de América Latina. El Acuerdo es fruto de un lento proceso negociador iniciado de manera oficial el 18 de octubre de 2012 con la formalización de una Mesa de Conversaciones cuya primera reunión tuvo lugar en Oslo (Noruega) para desarrollarse a partir de ahí en la Habana (Cuba). Finalmente, el Gobierno Nacional (con el Presidente Jesús Santos a la cabeza) y las FARC-EP suscriben el Acuerdo final, después de la inclusión de la modificaciones en el Acuerdo de paz previo suscrito el 24 de agosto de 2016, tras el rechazo en referéndum el 2 de octubre de ese año, lo que obligó a introducir cambios (poco sustanciales) que concluyeron en el denominado Acuerdo final.

El contexto en el que se desarrollan las conversaciones es la consecuencia de un "doble estancamiento doloroso" en que se encuentra el conflicto a la altura de 2012, entendido como "aquella tesitura por la cual ninguna de las partes considera que continuar en el marco de un conflicto resulta favorable para sus intereses" (Ríos, 2017: 598). Así en 2012 se llega al convencimiento por parte del Gobierno Nacional de la imposibilidad de una solución militar final contra la FARC-EP, tras años de aplicación de la Política de Seguridad Nacional y de un costo de más de 12.000 millones de dólares. Por el lado de la guerrilla, bajo una correlación de fuerzas desfavorable, encuentran en 2012 imposible la conquista del poder político por medio de la luchar armada, pero que a pesar de la pérdida de varias figuras destacadas en enfrentamientos con el ejército y de una disminución de su presencia en el territorio, conservaba en 
2012 más de 8.000 combatientes y una significativa implantación en las zonas tradicionales de la conflictividad armada en Colombia. Aspecto reconocido por el propio $\mathrm{PND}^{3}$.

La firma del Acuerdo final supone un éxito dado lo longevo de un conflicto histórico y enquistado, producto de una fase de la violencia armada en Colombia marcada por un doble proceso de "narcotización y periferialización" (Ríos, 2016). A pesar del debilitamiento de las FARC-EP, dicha organización lleva a cabo una táctica de repliegue guerrillero hacia territorios selváticos, mal comunicados, fronterizos y de escasa presencia estatal, zonas de arraigo guerrillero histórico y de importante presencia de los cultivos ilícitos relacionados con el narcotráfico, principalmente de cultivo de coca, que explican una geografía del conflicto más hostil para el ejército colombiano y que explican la resilencia de las FARC-EP y el enquistamiento territorial de la violencia armada en Colombia (PND, 2015 y Ríos, 2017).

El texto del Acuerdo final entiende que el fin del conflicto supone la apertura de una fase de transición del país hacia una nueva etapa de superación de tres problemas históricos que dieron lugar a la violencia armada que sufrió Colombia: el problema de la propiedad de la tierra en favor del campesinado, el abandono de las zonas rurales del país, de las comunidades campesinas y demás minorías sociales ${ }^{4}, \mathrm{y}$, finalmente, la escasa presencia del Estado en una parte importante del territorio nacional.

La descripción del contexto y de los marcos en los que se desarrollaron las conversaciones de paz, permiten entender el contenido de un Acuerdo, que en relación a las medidas económicas contempladas, pasa a analizar el texto.

\section{La solución al problema del campo en el Acuerdo de Paz}

Las medidas referidas a la solución del problema de la tierra a las que hace referencia el Acuerdo de paz, se contienen principalmente en el Punto 1 Hacia un Nuevo Campo Colombiano: Reforma Rural Integral y, en menor medida, en el Punto 3 Fin del Conflicto y Punto 4 Solución al Problema de las Drogas Ilícitas.

Ya desde la introducción, el Acuerdo de paz plantea los criterios centrales por los que se debe regir dicha política. Así se establece que Colombia "da inicio a una fase de transición que contribuya a una mayor integración de nuestros territorios" (Acuerdo de paz, 2016: 6), reconociendo como primer criterio la superación del déficit histórico que sufren las zonas rurales del país (Aguilera, 2010).

\footnotetext{
${ }^{3}$ Ver PND (2015: 40).

${ }^{4}$ Ver Acuerdo final (2016: 3 y 6).
} 
En la introducción el texto hace un reconocimiento expreso de "las necesidades, particularidades económicas, culturales y sociales" (Acuerdo de paz, 2016: 6), de las comunidades rurales, para lo cual se insta a llevar a cabo políticas que reconozcan el alcance estructural del problema de las zonas rurales y las comunidades campesinas, al requerir para su superación medidas elaboradas desde enfoques integrales, territoriales y de género.

Seguidamente, el texto reconoce que "La participación ciudadana es el fundamento de todos los acuerdos que constituyen el Acuerdo", por lo que toda medida debe contemplar el principio de participación social "en la planeación, la ejecución y el seguimiento de los planes y programas" (Acuerdo de paz: 2016: 7), con lo que junto a las diferencias territorial, el problema de la participación política del conjunto de la ciudadanía, en especial de la población campesina, sería el segundo déficit a superar en la Colombia que surge del posconflicto armado.

Junto a los anteriores, el Acuerdo final hace referencia a aspectos de inspiración ecoterritorial con referencias al concepto de buen vivir, al plantear que los acuerdos contemplados deben "garantizar la sostenibilidad socioambiental" (2016: 6), así como la superación de la brecha territorial entre el campo y la ciudad crea "las condiciones de bienestar y buen vivir para la población rural" (2016: 7).

El Punto $1^{5}$ contiene el acuerdo "Reforma Rural Integral", término con el que se pretende "contribuir a la transformación estructural del campo, cerrando las brechas entre el campo y la ciudad" (Acuerdo de paz: 2016: 7). Al emplear el término "estructural" se hace un reconocimiento a como el acceso de la propiedad de la tierra es la garantía del pleno disfrute de los derechos de la población campesina.

El Punto $3^{6}$ contiene el acuerdo de "Cese al Fuego y de Hostilidades Bilateral y Definitivo y la Dejación de las Armas", dentro del que se contemplan las medidas denominadas "Reincorporación de las FARC-EP a la vida civil -en lo económico, lo social y lo político- de acuerdo con sus intereses", de la que se pone de manifiesto la relación que establece el texto entre la desmovilización de las FARC-EP y el impulso de medidas de inserción económica y laboral de los ex combatientes que se acogen a la desmovilización. Al igual que la reforma agraria, el objetivo de la reinserción se asocia a medidas económicas que remiten a un modelo de cooperativismo agrario denominado ECOMÚN (Economías Sociales del Común) (Ríos, 2017).

El Punto $4^{7}$ dedica su artículo 4.1 al Programas de sustitución de cultivos de uso ilícito. Debido al alto rendimiento económico provenientes del cultivo

\footnotetext{
${ }^{5}$ Acuerdo de paz (2016: 10-34).

${ }^{6}$ Acuerdo de paz (2016: 57-97).

7 Acuerdo de paz (2016: 98-103).
} 
de la coca y su integración en las cadenas de procesamiento del narcotraficante, los estímulos que desincentiven la demanda y la producción cocalera son de gran importancia en la resolución de un conflicto, que ha tenido en el control de los enclaves cocaleros y sus rutas, escenarios centrales de la violencia armada dado el alto valor agregado de dichos territorios. Esto permite entender como la política de sustitución de cultivos aparece como prioritaria para el desarrollo exitoso del Acuerdo de paz así como para la seguridad pública del país, siendo necesarias medidas que desincentiven la producción de cultivos ilícitos y su sustitución por economías legales.

\subsection{La Reforma rural integral (RRI)}

El primer punto delAcuerdo final se centra en la modificación de la estructura de la propiedad de la tierra, con lo que se reconoce la relación existente entre la violencia política que sufre Colombia con el problema de la propiedad de la tierra. De esta forma el texto asume una de las reivindicaciones históricas de las FARC-EP, guerrilla de claro predominio campesino e importante presencia en las zonas rurales del país (Pizarro, 2011 y Ríos, 2017b).

El conflicto armado en Colombia hunde sus raíces en las guerrillas rurales que aparecen en dicho país en la década de los treinta del Siglo XX, y en el caso de las FARC, de las formadas en la región de Marquetalia en 1964 (Prieto, 1990), lo que permite entender la prioridad concedida durante el proceso negociador al debate de la tenencia de la tierra.

Esta conflictividad histórica descansa en las consecuencias sociales que hasta el día de hoy ha generado en Colombia el desigual acceso a la tierra, como atestigua el significativo proceso de concentración de la propiedad, que para el Programa de Naciones Unidad para el Desarrollo (2011) establece como el 1,1 por ciento de la población total del país es propietaria del 50 por ciento de la superficie de tierra, lo que hace de Colombia uno de los países más desiguales del mundo, tal y como se deriva de la CEPAL (2016), medido tanto por su coeficiente Gini según distribución de la renta $(0,54)$ como por el coeficiente Gini según distribución de la tierra $(0,85)$ (PND, 2015).

Con la RRI se pretende la transformación estructural del campo como "contribución central a la construcción de una paz estable y duradera", entendiendo por transformación estructural "el acceso progresivo a la propiedad rural de quienes habitan en el campo", reconociendo la función social de la propiedad como "el uso adecuado de la tierra" (2016: 10).

Definido el objetivo de la RRI, el texto plantea que la transformación estructural de la realidad rural colombiana debe contemplar los principios de: equidad, igualdad y democracia. De esta forma el acceso a la propiedad de la tierra se vincula al logro del bienestar de la población rural, la igualdad de 
género, la sostenibilidad y preservación del territorio campesino y la efectiva participación, definidos como el logro del "bienestar y buen vivir: [entendido como la] erradicación de la pobreza y la satisfacción plena de las necesidades de la ciudadanía de las zonas rurales" (Acuerdos de paz, 2016: 12).

El Acuerdo de paz concede gran importancia a la resolución del problema del "restablecimiento de la propiedad a aquellas personas que fueron desposeídas de la misma de manera ilegal durante el conflicto", restableciendo el derecho a la propiedad a las "víctimas del desplazamiento y del despojo" forzado de la tierra, pretendiendo con ello evitar la creación de condiciones futuras de regreso al conflicto armado (Acuerdo de paz, 2016: 13). Sin embargo, este principio de restablecimiento se vincula al no cuestionamiento del "derecho constitucional de la propiedad privada" (2016: 13) lo que puede derivar en un futuro proceso de judicialización de los reclamos de restitución de la propiedad y a la posible paralización de la efectividad de la devolución de la propiedad agraria a los desplazados por el conflicto.

De lo analizado hasta el momento, se desprende porque el objetivo del acceso y el uso a la propiedad de la tierra y la construcción de una economía campesina en los territorios marcados por el conflicto (principio de priorización), no deben descansar exclusivamente en el mercado. Es por esta razón que el Acuerdo de paz plantea una serie de instrumento desde los que garantizar la RRI, siendo el principal el establecido en el Punto 1.1.1 denominado "Fondo de Tierras para la Reforma Rural Integral", actor al que se encomienda el propósito de "lograr la democratización del acceso a la tierra en beneficio de los campesinos" (Acuerdos de paz: 2016: 14).

El Fondo de Tierras tendrá un carácter permanente y se dotará durante sus primeros 12 años de 3 millones de hectáreas, a las que se les reconoce el carácter de "tierras inalienables e inembargables", las cuales se distribuirán de manera gratuita por un periodo de 7 años (2016: 17). Las tierras adscritas al Fondo provendrán principalmente de procesos de compensación, adquisición $\mathrm{y}$, en menor medida, de expropiación, consecuencia de la importante protección que la propiedad privada tiene dentro del ordenamiento jurídico colombiano (Carvajal y Vicente, 2011), contemplando principalmente tierras que se encuentran fuera de la explotación dentro del mercado agrícola, lo que hace pensar que una parte de las tierras más productivas quedarán fuera del Fondo. Esta última circunstancia, obliga a establecer otros mecanismos de acceso a la propiedad de la tierra mediante subsidios parciales o integrales, líneas de créditos y otro tipo de subsidios dirigidos a su compra por parte del campesinado beneficiario, que junto a lo previsto al Fondo de Tierras, hacen de la de la propiedad privada la forma prioritaria de acceso a la tierra, frente a otras instituciones como el usufructo o la asignación de otro tipo de derechos de uso. 
La RRI plantea el acceso a la propiedad de la tierra en favor de trabajadores del campo sin tierra o "con tierra insuficiente" con la clara pretensión de crear una economía campesina basada en la pequeña y mediana propiedad rural de base familiar, designadas como Zonas de Reserva Campesina (ZRC) y cuya producción se dirigiría principalmente al autoconsumo de dichas zonas y al mercado interno formado en las zonas urbanas. Este es el objetivo de los Programas de Desarrollo con Enfoque Territorial (PDET), instrumentos de políticas de oferta dirigidos a fijar población rural en el territorio campesino marcado por el conflicto a través de las ZRC, que persiguen la formación de una pequeña propiedad rural viable económicamente.

Los PDET priorizarán su actividad en las "regiones abandonas y golpeadas por el conflicto" (Acuerdo de paz, 2016: 22), cuyo objetivo principal es crear las condiciones para la, hasta ahora, inexistente inserción de dichas zonas en el mercado interno, debido a la falta de infraestructuras, medios para la adecuación de tierras y de servicios públicos, déficits que se pretenden superar por medio de la puesta en práctica de diferentes Planes Nacionales detallados en el Punto 1.3 "Planes Nacionales para la Reforma Rural Integral", que hacen del Gobierno Nacional el actor principal en la puesta en práctica de las medidas económicas contempladas en el Acuerdo de paz.

Junto a las políticas de oferta mencionadas, se refleja la preocupación por la viabilidad económica de la pequeña producción campesina por su insuficiente escala y poca productividad, incapaces, por un lado, de crear economías locales y, por otro, de competir en el mercado de las zonas urbanas. Para conseguir el objetivo de la integración campo/ciudad, se plantean políticas de demanda (Puntos 1.3.3.3 y 1.3.3.4) que descansan principalmente en la generación de subsidios a la producción y al acceso de bienes de capital (semillas, tecnología, capacitación, créditos). Con ello se persigue la creación de mercados capaces de generar ingresos suficientes para una población rural víctima del conflicto y atraída por el atractivo de la alta productividad e ingresos provenientes de los cultivos ilegales.

\subsection{Las Economías Sociales del Común (ECOMÚN)}

El otro de los acuerdos que incluye menciones expresas al problema de las zonas rurales corresponde al Punto 3 "Fin del conflicto". El Punto 3 contempla dos apartados, el 3.1.4 que hace mención al acuerdo de cese al fuego y de hostilidades por medio de la puesta en práctica de las Zonas Veredales Transitorias de Normalización (ZVTN) y los Puntos Transitorios de Normalización (PTN); y, en segundo lugar, el apartado 3.2 denominado "Reincorporación de las FARC a la vida civil -en lo económico, lo social y lo político- de acuerdo con sus intereses" (2016: 68), donde aparecen las acciones 
más directamente relacionadas con políticas de carácter económico y laboral relacionadas con el campo.

Si bien las acciones previstas para la reincorporación de los miembros de las FARC a la vida civil, se contemplan sobre todo a través de medidas ligadas a la participación política e institucional de la guerrilla ya convertida en partido político, también se mencionan toda una serie de acciones relacionadas con la inserción laboral, social y económica de los excombatientes de las FARC-EP a la vida civil.

El Punto 3.2.2 "Reincorporación económica y social", es el artículo encargado de definir las medidas y acciones de carácter económica y laboral dirigidas al logro de "la reincorporación colectica económica y social" de los excombatientes (Acuerdo de paz, 2016: 75), objeticos que se encargan a la entidad denominada Economías Sociales del Común (ECOMÚN).

ECOMÚN aparece como el actor encargado de impulsar proyectos que promuevan de forma colectiva la reincorporación económica de los integrantes de las FARC-EP desmovilizados, concediendo el texto una mayor relevancia a la canalización de proyectos colectivos que los individuales, también reconocidos, pero con un grado de atención menor que aquellos que contemplen una dimensión comunitaria.

Para el cumplimento de los objetivos, ECOMÚN se dotará de un Fondo económico con el que financiar programas y proyectos colectivos de carácter productivo que serán verificados por la Consejo Nacional de la Reincorporación (CNR), organismo compuesto por dos miembros del Gobierno y dos de las FARC, Fondo que será provisto por recursos provenientes del presupuesto gestionado por el Gobierno Nacional.

La identificación de lo que el Acuerdo de paz denomina "programas y proyectos productivos sostenibles", se realizará a través de la confección de un censo socioeconómico cuyo objetivo es suministrar información detallada sobre aspectos sociales, laborales y de necesidades económicas de los miembros de las FARC-EP en proceso de desmovilización. El contenido del censo corresponderá al CNR y tendrá lugar dentro de los sesenta días siguientes al inicio de las ZVTN, siendo encomendada su realización y custodia de la información recogida en el censo a la Universidad Nacional de Colombia.

Cada integrante de las FARC-EP en proceso de reincorporación que desarrolle un programa productivo, bien sea colectivo o de forma individual, tendrá derecho por una vez, a la percepción de un apoyo económico por la suma de ocho millones de pesos (aproximadamente, 2600 euros).

Junto a ECOMÚN o el apoyo a proyectos de carácter individual, el Acuerdo final contempla un segundo tipo de medias de carácter económico basadas en políticas de transferencia directa de renta, en forma de retribuciones de carácter (cuasi) salarial, que se contemplan en forma de renta básica, asignación única de normalización y medidas por parte de la seguridad social. 
En relación a la renta básica, cada integrante de las FARC-EP en proceso de reincorporación, recibirá una renta básica mensual durante 24 meses, equivalente al 90 por ciento del Salario Mínimo Mensual Legal Vigente (SMMLV) o salario mínimo en Colombia, que supone aproximadamente 16 millones de pesos (aproximadamente, 5.200 euros), siempre que no haya otro vínculo contractual que genere ingresos tal y como de manera expresa plantea el apartado 3.2.2.7. La renta prevista puede beneficiarse de un incremento en función de la participación acreditada del integrante de las FARC-EP en proyectos educativos y de formación previstos en el proceso de reincorporación que pueden ser impartidos por ECOMÚN.

También se prevé una asignación a cada integrante de las FARCEP al momento de terminación de las ZVTN, asignación denominada de "normalización" equivalente a dos millones de pesos (aproximadamente, 650 euros), decisión que fue criticada por los sectores políticos contrarios al acuerdo, que sin embargo fue ya contemplada en 2005 en el proceso de desmovilización de organizaciones paramilitares bajo la presidencia de Álvaro Uribe (Ríos, 2017).

En cuanto a los pagos por concepto de seguridad social en salud y pensiones, serán garantizadas por el Gobierno Nacional durante 24 meses, extensibles a 36 meses, en caso de integrantes de las FARC-EP con enfermedades graves, rehabilitación de lesiones derivadas del conflicto, situaciones que deben estar acreditadas por la CNR.

Por último, y junto a las anteriores, se prevé la participación de los excombatientes en planes programas sociales relacionados con la educación formal, la formación profesional, de reunificación familiar o protección de hijos integrantes de las FARC. Los planes que contarán con un tratamiento prioritario serán los relacionados con el acceso a la vivienda, a los que el Gobierno Nacional dará tratamiento especial, bien sea en autoconstrucción o provenientes de programas de ECOMÚN.

El acuerdo pone de manifiesto la importancia central que para el proceso de paz adquiere la desmovilización y las dificultades que la propias FARCEP prevén para la reincorporación de la antigua guerrilla en los enclaves que protagonizaron el conflicto armado. La inclusión en el texto de la expresión "de acuerdo con sus intereses" es interpretado por el Acuerdo final como "La reincorporación a la vida civil [de los excombatientes guerrilleros] será un proceso de carácter integral y sostenible, excepcional y transitorio que considerará los intereses de la comunidad de las FARC-EP en proceso de reincorporación" (2016: 68-69). Con ello la antigua guerrilla pretende trasladar a la letra del Punto 3 "la impronta comunitaria que las FARC han conferido al proceso de negociación", relacionada con "la reincorporación colectiva y a la promoción de mecanismos de inserción económica y laboral cooperativos 
y solidarios con marcada impronta rural" (Ríos, 2017: 607), propia de una organización de predominio campesino como son las FARC.

\subsection{Punto 4.1 Programas de sustitución de cultivos de uso ilícito}

El proceso de narcotización es una de las principales características del conflicto armado en Colombia dada la relación de los cultivos cocaleros con la violencia armada y su evolución más reciente (Herderson, 2010; Pécaut, 2008 y Ríos, 2016). Para el Acuerdo de paz la solución de las drogas ilícitas pasa por un programa integral de sustitución de cultivos ilícitos, que en la actualidad alcanzan las 188.000 Ha (UNODC, 2017), localizadas en gran parte en las regiones que han conformado la geografía del conflicto armado en Colombia como son los departamentos de Nariño, Putumayo, Cauca o Norte de Santander (Ríos, 2016b).

El artículo 4.1 reconoce la importancia que para la construcción de la paz en Colombia tiene la sustitución de cultivos de uso ilícito, y como dicho objetivo, se relaciona en gran parte con la puesta en práctica de alternativas económicas. Para el Acuerdo de paz el conflicto depende de generar "condiciones materiales e inmateriales de bienestar y buen vivir (...) para las comunidades campesinas en situación de pobreza", cuya subsistencia se relaciona en gran parte de los ingresos que provienen de dichos cultivos (2016: 102).

Por ello, el Acuerdo final presenta medias y acciones dirigidas a dar alternativas al bienestar de las poblaciones campesinas afectadas por el conflicto y por la economía derivada de las drogas ilícitas "como parte de la transformación estructural del campo que busca la RRI" (Acuerdo de paz, 2016: 100).

Hacer efectiva la RRI en lo que respecta a la sustitución de cultivos de uso ilícito, es la función de los Planes Integrales de Sustitución y Desarrollo Alternativo (PNIS), a los que se les encomienda la puesta en práctica de alternativas que logren el compromiso de las comunidades de avanzar en los procesos de sustitución voluntaria, siendo éste último "un factor fundamental para el logro de los objetivos" (Acuerdo de paz, 2016: 100) y que se orienta, principalmente, en torno a diferentes principios.

En primer lugar, el PNIS se contemplan como parte integrante de la RRI, considerado como un componente de la transformación del campo colombiano al que la reforma rural aspira, lo que le lleva a ser parte de los enfoques territoriales previstos en los PDET. Se reconoce, por tanto, la aplicación a las comunidades cocaleras de los beneficios y medidas contempladas en el acuerdo para la RRI, tal y como prevé el artículo 4.1.3 "Descripción y elementos del PNIS", al hacer extensivo a los mismos de toda la política de acceso y formalización de la propiedad, adecuación de tierras, vivienda, créditos, 
subsidios, políticas de acceso al mercado y de provisión de bienes y servicios públicos contemplados en el punto 1 .

En segundo lugar, y junto a la incorporación del programa de sustitución de cultivos en la RRI, el otro eje rector del acuerdo es el principio de sustitución voluntaria, punto por el que expresamente el Estado colombiano renuncia a los mecanismos de erradicación forzosa y asume el "principio de sustitución voluntaria, sostenible, participativa, diferencial y adecuada a las necesidades y demandas de las comunidades rurales" (Acuerdo de paz, 2016: 103), por las que se tendría que poner fin a las prácticas gubernamentales basadas en la erradicación de cultivos ilícitos por aspersión con glifosato (Ríos, 2017).

En relación a los beneficiarios del PNIT y una vez hechos el compromiso con la sustitución del cultivo ilícito, el acuerdo contempla la actuación en favor de tres colectivos concretos: cultivadores de coca y sus familias, recolectores y comunidades campesinas de zonas cocaleras.

En primer lugar, medidas dirigidas a familiares de los cultivadores cocaleros, fundamentalmente, centradas en garantizar la asistencia alimentaria inmediata por medio de bonos hasta por un año, el establecimiento de huertas caseras y provisión de insumos necesarios y proyectos de generación de ingresos económicos rápidos, en primera instancia relacionados con la formación de mercados locales campesinos por las mismas comunidades cocaleras o economías relacionadas con labores de sostenibilidad medio-ambiental; y, en segunda instancia, favoreciendo el acceso a segmentos de demanda de los núcleos urbanos o de la demanda internacional.

En segundo lugar, se encuentran como beneficiarios los colectivos de recolectores asentados o temporales, a los que se le aplicarán las medidas de garantía de asistencia alimentaria inmediata hasta un año contempladas para los cultivadores, así como la opción a empleos ligados a obras comunitarias y otras fuentes de empleo que emanen de la puesta en práctica de la RRI en su comunidad, además la extensión de las líneas de créditos y concesión de subsidios en los mismos términos de los previstos en el artículo 1.1.3.

El tercer grupo de beneficiarios, estará formado por los miembros de la comunidad que habita los territorios afectados por los cultivos ilícitos, los cuales podrán acceder a las ofertas de empleo, de política de bonos y subsidios previstos para los beneficiarios anteriores, a lo que se unen políticas educativas en favor de la población escolar, la mujer integrante de dichas comunidades cocaleras y adultos mayores.

El punto 4.1.3 termina con la disposición de actuaciones en infraestructuras y adecuación de los cultivos ilícitos en tránsito a la economía legal, relacionados con políticas de sustitución por usos medio-ambientales y comunales que permitan algo para el Acuerdo muy importante, como es el cierre de la frontera agrícola y la presencia del Estado en zonas hasta ahora fuera de su control efectivo. 


\section{El modelo de desarrollo contemplado en el Acuerdo de paz}

El predominio de la cuestión agraria es la nota característica de las medidas y acciones económicas contempladas en el Acuerdo de paz, texto que queda determinado por la visión política de un conflicto que se entiende derivado de la histórica reivindicación campesina por el acceso a la tierra. A pesar de éste determinante político, el texto del Acuerdo final dibuja una concepción cercana a la Economía del desarrollo favorable a la creación de una economía campesina, insertada en un sistema agrario mercantil basado en la pequeña propiedad familiar bajo forma de cooperativa agrícola.

La garantía del acceso a la pequeña propiedad por parte del campesinado sin tierra se contempla como la medida principal para el logro de tres objetivos: la paz, el bienestar económico y social del campesinado y el tránsito a economías legales por parte del campesinado cocalero.

El éxito del modelo descansa, en un primer momento, en la generación de un mercado local campesino con el que autoabastecer la demanda de las zonas rurales, en especial, las marcadas por el conflicto, pero con la intención posterior, de orientar los resultados de la RRI hacia la integración campo/ciudad gracias a la capacidad de abastecer una parte de la demanda interna de alimentos de las zonas urbanas por medio de la producción de las nuevas economías campesinas resultado del proceso de paz. La RRI sería por tanto, la constructora de una economía campesina, desde la que favorecer la acumulación del sector agrario nacional, con predominio del intercambio mercantil.

Si por el lado de la demanda se entiende que son los mercados locales y el mercado nacional de las grandes ciudades el motor (la demanda externa apenas es contemplada), por el lado de la oferta, el Estado aparece como el protagonista en la asignación de bienes y servicios por encima del mercado, con lo que el modelo de consumo de las comunidades campesinas quedará bajo el predominio de bienes de consumo colectivo provistos desde políticas públicas provenientes del Gobierno Nacional.

A pesar de las menciones expresas de perseguir una "transformación integral del campo colombiano", la letra del Acuerdo final en cuanto a medidas económicas se entiende, parte de la idea del campesino sin tierra o con tierra insuficiente como el beneficiario principal, el reconocimiento de diferentes tipos de propiedad (cooperativa o social) dentro de una economía de mercado con predominio de la propiedad privada, donde las zonas afectadas por el conflicto se declaran como las áreas geográficas de actuación prioritaria, encomendándose al Estado, entendido éste como el Gobierno Nacional, la viabilidad y puesta en práctica de la RRI. 


\section{EI Plan Nacional de Desarrollo 2014-2018: Todos por un nuevo país ${ }^{8}$}

El PND se define como el texto que "plasma la estrategia política del gobierno del presidente Juan Manuel Santos" (2015: 24) durante el periodo 2014-2018, lo que remite al momento final del proceso de negociación con las FARC-EP y posterior suscripción del Acuerdo final. Para el PND "la materialización de los eventuales acuerdos de paz (...) se soportarán sobre las políticas y estrategias que este Plan Nacional de Desarrollo le propone al país para los próximos años" (2015:25), constituyéndose como el principal texto desde el que analizar la política económica y social del Gobierno Nacional en la fase del posconflicto, texto central para entender cómo el Gobierno colombiano piensa poner en práctica las medias contempladas en el Acuerdo de paz.

Para el PND, el posconflicto armado abre un escenario con el que liberar recursos que sitúen a Colombia en una senda de "paz, equidad y educación", principios que conforman un "círculo virtuoso" de desarrollo del país (PND, 2015: 23).

\subsection{La transformación del campo y la noción de desarrollo previsto en el PND}

Para el PND la paz constituye una oportunidad histórica, cuando considera que “(...) Colombia tiene ahora una oportunidad única de romper con su legado histórico del conflicto. Con la consolidación de la paz las posibilidades de desarrollo de Colombia en otras dimensiones se potencian" (2015: 24). La relación entre los problemas de ordenación social y productiva del campo y la conflictividad social en Colombia tiene que ver "con la falta de acceso a la tierra, inseguridad jurídica y conflictos de uso del suelo" (2015: 417), en un país donde "sólo se utiliza un 24 por ciento de las tierras aptas para el cultivo, (...) el 75, 6 por ciento de la propiedad rural tienen menos de 5 hectáreas y la pobreza extrema rural es 3,5 veces mayor que la urbana" (2015: 417-418), lo que explica la urgente necesidad de la transformación del campo colombiano.

La concepción de desarrollo de la que parte el PND queda definida en su apartado primero dedicado a la introducción, al entender el desarrollo como "lograr la modernización del país". Dicho objetivo que se remite a la mención expresa de autores como Besley, Persson y Fukuyama y su concepción del "Estado moderno y Estados efectivos"; continúa con la puesta en valor de experiencias internacionales como las del modelo educativo chileno (2015: 24-

\footnotetext{
${ }^{8}$ Aprobado el 9 de junio de 2015 por la Ley 1753, por la que se expide el Plan Nacional de Desarrollo 2014-2018 "Todos por un nuevo país". Disponible en web: http://www.achc.org.co/ documentos/prensa/LEY-1753-15\%20Plan\%20Nacional\%20de\%20Desarrollo\%202014\%20-\%20 2018.pdf
}

Araucaria. Revista Iberoamericana de Filosofía, Política y Humanidades, año 20, no 39. Primer semestre de 2018. Pp. 365-387. ISSN 1575-6823 e-ISSN 2340-2199 doi: 10.12795/araucaria.2018.i39.18 
25), conceptos como el de capital social de Putnam ${ }^{9}$ (2000), o la concepción de crecimiento económico en torno a la teoría de las ventajas competitivas de Porter ${ }^{10}$ (2012). Ello situaría el modelo de desarrollo contemplado por el PND como parte de las corrientes que se reclaman de "las nuevas teorías del crecimiento" contemplados por Kaldor (1961), que introducen al enfoque modernizador la importancia de los factores endógenos de acumulación de naturaleza local derivados de la "nueva economía geográfica" de Krugman (1997).

Al describir los principios del modelo de desarrollo contemplado en el PND, se puede entender mejor cual es la concepción que el Gobierno Nacional tiene del problema de la cuestión rural en Colombia, la cual parte de una doble perspectiva.

En primer lugar, el desarrollo como una relación entre atraso y modernidad. El PND apoya la idea de la existencia de estadios de desarrollo del cual se desprenden niveles diferenciados y de jerarquía espacial dentro del país al hablar de "3 Colombias", donde la primera "es la Colombia urbana, moderna, productiva (...). La segunda, cuenta con un desarrollo incipiente (...). La tercera Colombia hace referencia a la Colombia rural, especialmente en las zonas más apartadas (...)" (PND, 2015: 37).

En segundo lugar, el desarrollo entendido como crecimiento económico. El desarrollo como producto de una relación jerárquica entre la sociedades y el espacio, se reproduce en -para el PND- en las relaciones entre la escala nacional y la economía mundial (entendida indistintamente como globalización), de tal forma que, por un lado, la modernización del campo colombiano será producto de su relación con el progreso de las ciudades, mientras que por otro, la modernización de Colombia como nación será la consecuencia de su inserción exitosa en la globalización, haciendo del desarrollo "una estrategia de competitividad central para que Colombia se articule a la economía mundial (...) y alcanzar así mayores niveles de crecimiento" (PND, 2015: 27).

De esta forma, el conflicto en Colombia es visto como una carencia de desarrollo producto del atraso de las zonas rurales del país, el cual se produce por la falta de adaptación del campo colombiano a las condiciones favorecedoras del progreso existente en las zonas urbanas. De aquí se puede extraer la noción de transformación del campo entendido como el proceso de conexión de las zonas atrasadas del país (las rurales) con las zonas desarrolladas y productivas de Colombia (las zonas urbanas). Se traduce así una relación jerárquica entre economía mundial, zonas urbanas y zonas rurales, por la que el desarrollo será consecuencia de la articulación de las zonas atrasadas con las más modernizadas vía relaciones económicas basadas en el mercado.

\footnotetext{
${ }^{9}$ Ver Capítulo VI “Movilidad social” (PND, 2015).

${ }^{10}$ Ver Capítulo X "Ejes regionales" (PND, 2015).
} 


\subsection{La transformación del campo colombiano prevista en el PND}

El concepto de "transformación del campo" se contempla en el Capítulo VII del Tomo I del PND ${ }^{11}$, noción que se relaciona con la interpretación del conflicto armado en Colombia como una consecuencia del marco ambiental en el que se desenvuelven la sociedad rural colombiana, el cual debe modificarse a través de la transformación del campo colombiano.

Al igual que en el Acuerdo de paz, los problemas de las zonas rurales "están relacionados con la falta de acceso a la tierra, la inseguridad jurídica y conflictos en el uso del suelo" (PND, 2015:417), para lo que se necesita una política dirigida a "un desarrollo rural integral (...) desde el que cerrar las brechas sociales" (2015: 49), concepto también contemplado en el Acuerdo final.

Esta visión de la transformación del campo como necesaria para alcanzar un paz estable y duradera, depende para el PND del éxito económico del sector agrícola, entendido como el logro de "crecimiento y la competitividad de las actividades económicas rurales (...). Esto permitirá que en el mediano y largo plazo los habitantes del campo puedan vivir dignamente" (PND, 2015: 422).

Con la transformación del campo se pretende conseguir "el ejercicio de actividades económicas competitivas y sostenibles con la tranquilidad de contar con una institucionalidad que los represente" (2015: 422), cuyo objetivo se define como "la ampliación de la clase media rural a través de una apuesta de inclusión productiva de los campesinos", lo cual permita "cerrar las brechas urbano-rurales y sentar las bases para la movilidad social", mediante una ordenación "del territorio rural buscando un mayor acceso a la tierra por parte de los productores agropecuarios sin tierra o sin tierra suficiente" (2015: 422).

Definida la visión y los objetivos que se pretenden, el PND concreta las medias con las que alcanzar la propuesta de desarrollo rural integral, que se resumen en las dirigidas al acceso de la tierra, el cierre de las brechas urbano/ rurales, la ampliación de la clase media rural, medidas de aumento de la competitividad rural y la creación de un nuevo marco institucional al servicio de las anteriores.

En relación al acceso de la tierra, el PND contempla medidas y acciones que tienen como objetivo el acceso a la propiedad de la tierra o la restitución de la propiedad a aquellos campesinos que fueron desposeídos ilegalmente de ella en el contexto del conflicto armado. Se apuesta por mecanismos de acceso a la tierra en favor de la "población rural de escasos recursos que carece de tierra o la tiene de forma insuficiente. (...) y continuar con la política de restitución de tierras en favor de las víctimas" (PND, 2015: 423).

\footnotetext{
11 Ver PND, 2015 (pp: 415-446).
} 
Para ello, el Estado se compromete a la creación de fuentes de tierra suficientes, por medio de procesos de desamortización y paso al mercado reglado de tierras ilegales o de usos que se entienden no productivos (entre ellos zonas forestales), así como -de manera excepcional- la posibilidad de la compra a cargo del Estado de tierras si se observa que la provisión de tierras en insuficiente.

Con estos mecanismos se pretende la creación de una base de nuevos campesinos propietarios, que apoyados por políticas de provisión de bienes colectivos (infraestructuras, vivienda, educación, sanidad y apoyo técnico), deben configurar una clase media propietaria, que en el ámbito rural, sea capaz de dirigir un proceso modernizador similar al experimentado -según describe el PND- en las zonas urbanas. Por último, se establecen líneas de créditos, de apoyo a la distribución, asociacionismo empresarial y necesidades logísticas, con las que se busca aumentar la capacidad competitiva del campo colombiano y así conectarlo con la demanda de las zonas urbanas y el mercado internacional.

A diferencia del Acuerdo de paz, el PND no contempla acciones del Estado de apoyo directo a la producción, medidas de salario diferido u otros tipos de transferencias de renta, acercándose en sus propuestas a los planteamientos y recomendaciones recogidos en el documento de la OCDE (2015), al recoger el principio de subsidiariedad del "Estado moderno, más transparente, eficiente y eficaz (...) que asegure tanto mercado como sea posible y tanto Estado como sea necesario" (2015: 28).

\section{Conclusiones finales}

Con base a lo expuesto se pueden apreciar diferentes definiciones del concepto de transformación del campo colombiano y la noción de desarrollo rural integral contemplado en el Acuerdo de paz y el PND. Si bien ambos, comparten el problema del acceso a la tierra por parte del campesinado pobre como la prioridad social a resolver en el escenario del posconflicto armado, de lo analizado se aprecian cinco niveles de contradicción entre ambos documentos.

En primer lugar, aparecen las contradicciones derivadas de las relaciones entre economía mundial y economía nacional, entre la RRI que toma como prioridad la demanda interna y el PND que se orienta hacia la demanda exterior. El "desarrollo rural integral" del que habla el Acuerdo de paz, prevé la promoción de una "economía campesina, familiar y comunitaria", cuya producción se dirige a "la promoción de mercados locales y regionales (...) y fomente la producción y el consumo de alimentos nacionales" (2016:34), lo que hace del mercado interno el motor de la transformación del campo colombiano para el Acuerdo de paz. Sin embargo, de la lectura del PND se desprende un 
modelo de desarrollo para el campo colombiano diferente, que toma como eje la "mejor inserción del mismo en la economía mundial" sobre los patrones de la teórica ortodoxa del comercio internacional: la apertura exterior, la explotación de las ventajas comparativas y el aumento de la productividad como criterio de competitividad.

Las políticas de competitividad y de mayor apertura de campo colombiano al mercado exterior que plantea el PND, contradicen en cierta forma la apuesta por la formación de economías territorialilzadas contemplada en las RRI, situación reconocida en el texto del Acuerdo final al manifestar que "el desarrollo rural integral se adelantará en un contexto de globalización y de políticas de inserción en ella por parte del Estado" (2016: 12), que descansa en la progresiva adaptación de las nuevas economías campesinas al mercado mediante dinámicas "competitiva e insertada en cadenas de valor agregado" (2016: 33). Excepto la anterior mención, todo lo tratado en Acuerdo de paz sobre esta importante realidad, se remite a fórmulas genéricas que instan al Gobierno Nacional para que "se eviten o minimicen los impactos negativos que supongan la internacionalización de la economía y la liberalización del comercio" (2016: 33), algo no contemplado en el texto del PND.

Surge un segundo aspecto no resuelto, relacionado con el tipo de articulación económica que debe seguirse para la superación de la brecha campo/ciudad, en un contexto donde la relación es, por un lado, de predominio exportador de los productos agrícolas nacionales y, por otro, el predominio de bienes importados para el consumo interno (OCDE, 2015). Según lo anterior, la economía colombiana tiende a la sustitución de los productos de las economías campesinas nacionales por bienes importados más baratos y mejor insertados en las cadenas de comercialización, realidad negativa que se pretende superar por parte del Acuerdo de paz estableciendo una articulación pública campo/ciudad que descansa en la puesta en práctica de un Plan Nacional para la promoción de la comercialización de la producción de la economía campesina, familiar y comunitaria, cuya medida central consistiría en "la implementación progresiva de un mecanismo de compras públicas para atender a las demandas de las entidades y programas institucional (...) que fomente la producción local" (2016: 31). Sin embargo, dicha práctica está prohibida por la Organización Mundial del Comercio (OMC) a través de la cláusula de igualdad de trato, la cual exige que la normativa nacional que afecta a las operaciones comerciales se aplique sin discriminaciones entre productos nacionales e importados, clausula vigente en los tratados de libre comercio (López, 2010).

Asimismo, el Acuerdo de paz prevé en su artículo 1.3.3.3 una política de subsidios y apoyo económico por parte del Gobierno Nacional al productor agrícola local, medida que sin embargo no es contemplada en el PND $^{12}$ el cual

${ }^{12}$ Leer Objetivo 4. Impulsar la competitividad (PND, 2014: 434 y 438). 
apuesta por políticas horizontales (no discriminatorias) y de oferta (suministro de bienes públicos), dirigidas a mejorar la capacidad competitiva de la producción local y su inserción exterior, que casa mejor con lo previsto en el artículo 1.3.3.6 del Acuerdo de paz, el cual viene a recocer que la viabilidad económica de las cooperativas campesinas vendrá dada a medio plazo por su capacidad competitiva. Hecho que convierte de facto al mercado como el principal asignador de bienes y servicios, con lo que se provocaría, a medio plazo, una posible expulsión de los productos de las economías campesinas nacionales de los mercados de las zonas urbanas en favor de alimentos importados.

En tercer lugar, destacan las dificultades que se pueden derivar de las necesidades de inversión pública que requiere la periferia rural existentes en un país fuertemente centralizado, donde el 85 por ciento del presupuesto público es gestionado por el Gobierno Nacional, el 5 por ciento los gestiona el Distrito Capital, el 5 por ciento los gestionan los 32 departamentos en los que se divide la administración regional del país, quedando el 5 por ciento restante para los más de 1.120 municipios colombianos (Ríos, 2017), en un país donde el 84,7 por ciento de su territorio está conformado por municipios totalmente rurales, en los que vive el 30,4 por ciento de la población del país y se concentra el 16,3 por ciento del empleo (PND, 2015: 417). Sin embargo, la importancia social y territorial del campo colombiano no corresponde a su peso económico que se ha reducido en la última década, consecuencia de su poca productividad y capacidad competitiva (OCDE, 2015 y CEPAL, 2016), lo cual profundiza el problema de pobreza y desigualdad social, realidad reconocida tanto por el Acuerdo final como por el PND. Tanto los PDET previstos en el Acuerdo final como las estrategias regionales previstas en el Tomo 2 del PND, plantean el fortalecimiento de los municipios en el diseño de las políticas públicas, que sin embargo en la práctica no suponen un proceso de descentralización de los recursos efectivos, al no dotarse el Gobierno Nacional de una reforma fiscal que asegure la suficiencia de los recursos y mejore la capacidad redistributiva, ni apueste por una descentralización del gasto en favor de los municipios rurales, tal y como ha puesto de manifiesto la Contraloría General de Colombia ${ }^{13}$. Hechos que pueden conducir a una ineficiente asignación de recursos entre las necesidades de la periferia rural y la concentración presupuestaria en el centro.

En cuarto lugar, se encuentran las dificultades de encontrar incentivos efectivos para el abandono de unos cultivos relacionados con actividades ilícitas pero altamente productivas y lucrativas, en comparación con las expectativas futuras de las producciones agrarias legales, pero de incierta viabilidad económica. La creación de economías campesinas alternativas a los cultivos ilegales cuenta con las dificultades de la falta de infraestructuras que liguen dichas producciones con sus mercados potenciales, realidad contraria a

\footnotetext{
${ }^{13}$ Ver apartado III (2015: 25).
} 
unos cultivos de la coca mejor insertados en las cadenas de comercialización controladas por el narcotráfico, de las que se derivan unos altos rendimientos productivos en relación a las economías locales previstas por el Acuerdo final y el PND, cuyos resultados requieren de tiempo para su implementación y resultados.

Como resumen de las anteriores, se podría concluir con una quinta consideración, relacionada con la existencia de modelos de desarrollo diferentes $\mathrm{y}$, en ocasiones contradictorios, entre la concepción de desarrollo entendido como buen vivir y bienestar incluida en el Acuerdo de paz y una visión más ortodoxa, que contempla el desarrollo como crecimiento económico definida en el PND. El Acuerdo final parte de un paradigma de desarrollo basado en el concepto de buen vivir, próximo a los paradigmas posdesarrollistas (Braña, Domínguez y León, 2016) el cual, a pesar de reconocer el carácter mercantil de las relaciones dentro del campo colombiano, se remite a la función social y ecológica de la propiedad, que plantea límites a la misma (sea nacional o extranjera) y que apuesta por una economía de mercado regulada y basada, en muchos aspectos, en economías cooperativas donde el asignador de bienes y servicios no corresponde enteramente al mercado. Concepción diferente a la del PND, el cual entiende el desarrollo como crecimiento económico, más cercano a los paradigmas modernizadores (Ramírez, 2017), donde el crecimiento económico es la consecuencia de los incrementos de productividad producidos por la dinámica competitiva, donde las brechas sociales y territoriales se verán superadas con la puesta en relación de la zonas menos avanzadas con las más desarrolladas, entendido como la relación campo/ciudad por un lado, y la relación mercado nacional/mercado global, por otro.

No obstante, el reconocimiento del problema del acceso a la tierra y de la pobreza y abandono del medio rural como uno de los causantes del conflicto en Colombia, supone un avance histórico y un reconocimiento a la raíz estructural de la violencia armada en dicho país. La dimensión del problema requiere de medidas y acciones dirigidas a la transformación del campo en Colombia sobre la base de la adopción de un modelo de desarrollo que sitúe la solución de la brecha campo/ciudad y la solución a la concentración de la propiedad de la tierra, desde una dimensión integral.

Aspectos que requieren de la superación de las contradicciones puestas de manifiesto, entre la noción de transformación del campo y de desarrollo rural integral existentes entre el Acuerdo de paz y la política económica del Gobierno Nacional, principal actor responsable en el éxito del actual escenario del posconflicto armado en Colombia. 


\section{Referencias bibliográficas:}

Acuerdo de paz (2016): Acuerdo final para la terminación del conflicto y la construcción de una paz estable y duradera, Oficina del Alto Comisionado para la Paz, Bogotá. Disponible en web: http://www. altocomisionadoparalapaz.gov.co/procesos-y-conversaciones/ Documentos\%20compartidos/24-11-2016NuevoAcuerdoFinal.pdf

Aguilera, Mario (2010): Las FARC: la guerrilla campesina 1949-2010, Arfo Editores, Bogotá.

Braña, Francisco Javier, Domínguez, Rafael y León, Mauricio (2016): Buen vivir y cambio de la matriz productiva. Reflexiones desde Ecuador, Friedrich-Ebert-Stiftung (FES-ILDIS), Ecuador. Disponible en web: http://library.fes.de/pdf-files/bueros/quito/12781.pdf

Carvajal Ossa, Manuela y Vicente Pérez, Vicente (2011): La expropiación en Colombia y su historia en la legislación colombiana. Dirigida por Diana Cecilia Velásquez Rendón. Monografía de grado presentada para optar por el título de Abogado, Universidad EAFIT, Escuela de Derecho, Medellín. Disponible en web: https://repository.eafit.edu.co/bitstream/ handle/10784/8097/Juliana_VicentePerez_Manuela_CarvajalOssa_2011. pdf? sequence $=2$

Comisión Económica para América Latina (2016): Panorama social América Latina, Santiago de Chile, CEPAL. Disponible en web: http://repositorio. cepal.org/bitstream/handle/11362/41598/4/S1700567_es.pdf

Contraloría General dela República de Colombia (2015): Análisis y evaluación del Plan Nacional de Desarrollo 2014-2018 "Todo por un nuevo pais", Contraloría General, Bogotá. Disponible en web: http://www. contraloria.gov.co/documents/463406/472376/Comentarios+al+Plan+ Nacional+de+Desarrollo+2014-2018.pdf/e1781355-35fb-45da-9eea$0 \mathrm{a} 12269 \mathrm{~d} 7778$ ?version $=1.0$

Henderson, James (2010): Víctimas de la globalización. La historia de cómo el narcotráfico destruyó a Colombia, Siglo del Hombre, Bogotá.

Kaldor, Nicholas (1961): Ensayos sobre desarrollo económico, Centro de Estudios Monetarios Latinoamericanos, México.

Krugman, Paul (1997): Desarrollo, geografía y teoría económica, Antoni Bosh, Barcelona.

Observatorio de Derechos humanos y Derecho Internacional Humanitario (ODHDIH). (s.f.): Sintesis de la violencia y la confrontación armada en Colombia, 1998-2012 y 2015, Bogotá. 
OCDE (2015): Revisión de la OCDE de las Políticas Agrícolas: Colombia 2015. Evaluación y recomendaciones de Política agraria. Disponible en web: https://www.oecd.org/countries/colombia/OECD-ReviewAgriculture-Colombia-2015-Spanish-Summary.pdf

Pécaut, Daniel (2008): "Las FARC: fuentes de su longevidad y de la conservación de su cohesión", Análisis Político, núm. 63, mayo-agosto, Bogotá, pp: 22-50. Disponible en web: https://doaj.org/article/26b274784 9794c06a2f958e738302426

Plan Nacional de Desarrollo (2015): Plan Nacional de Desarrollo 20142018: Todos por un nuevo país Tomos 1 y 2, Departamento Nacional de Planeación, Bogotá. Disponible en web: https://www.dnp.gov.co/PlanNacional-de-Desarrollo/Paginas/Que-es-el-Plan-Nacional-de-Desarrollo. aspx

Pizarro, Eduardo (2011): Las FARC (1949-2011). De guerrilla campesina a máquina de guerra, Norma editorial, Bogotá.

Porter, Michael E. (2012): Estrategia competitiva: técnicas para el análisis de los sectores industriales y de la competencia, Editorial Pirámide, Madrid.

Prieto, Alberto (1990): Guerrillas en América Latina, Ediciones Políticas, La Habana.

Programa de Naciones Unidad para el Desarrollo (2011): Informe sobre Desarrollo Humano. Colombia rural: Razones para la esperanza, Naciones Unidas, Nueva York. Disponible en web: http://www.undp.org/ content/dam/colombia/docs/DesarrolloHumano/undp-co-ic_indh2011parte1-2011.pdf

Putnam, Robert (2000): The collapse and revival of American community, Simon \& Schuster, New York.

Ramírez Cendrero, Juan Manuel: (2017): “Límites y contradicciones del postdesarrollo como inspiración heterodoxa frente al desarrollo capitalista", Revista de Economía Crítica, n 24, segundo semestre. Disponible en web: http://www.revistaeconomiacritica.org/sites/default/ files/revistas/n24/JuanMRamirez_Critica-al-giro-postestructuralista.pdf

Ríos, Jerónimo (2016): "La narcotización del activismo guerrillero de las FARC y el ELN, 1998-2012”, Revista UNISCI, núm. 41, mayo, pp: $205-$ 234. Disponible en web: https:/www.ucm.es/data/cont/media/www/pag83486/UNISCIDP41-9RIOS.pdf

Ríos, Jerónimo (2016b): Breve historia el conflicto armado en Colombia, Los Libros de la Catarata, Madrid. 
Ríos, Jerónimo (2017): "El Acuerdo de paz entre el Gobierno colombiano y las FARC: o cuando una paz imperfecta es mejor que un guerra perfecta", Araucaria. Revista Iberoamericana de Filosofía, Política y Humanidades, Universidad de Sevilla, año 19, no 38. Segundo semestre, pp: 593-618. Disponible en web: https://revistascientificas.us.es/index.php/araucaria/ article/view/4020/3493

Ríos, Jerónimo (2017b): "Determinantes geográfico-políticos de la acción violenta guerrillera: un análisis de la concurrencia regional de guerrillas y paramilitares en el conflicto colombiano", Revista Española de Ciencia Politica, 44, pp: 121-149.

López Barreiro, Esther (2010): Regulación del comercio internacional: La $O M C$, Tirant lo Blanch, Valencia.

United Nations Office on Drugs and Crime (2016): Colombia. Monitoreo de territorios afectados por cultivos ilícitos, julio, Oficina de las Naciones Unidas contra la Droga y el Delito Oficina Colombia, Gobierno de Colombia. Disponible en web: http://www.biesimci.org/Documentos/ Documentos_files/Censo_cultivos_coca_2016.pdf 
